\title{
LE CODE FAIT-IL VRAIMENT LA LOI ? ANALYSE de LA PARTICIPATION POLITIQUe SUR LA PLATEFORME GiTHUB
}

\author{
Édouard BouTÉ ${ }^{1}$ et Clément MABI ${ }^{2}$
}

\begin{abstract}
À travers le cas de GitHub, plateforme numérique de " social coding » permettant la collaboration entre informaticien-nes et parfois utilisée comme espace de participation politique, cet article discute la théorie des « tiers espaces » qui suggère que les espaces non destinés à accueillir une telle participation peuvent favoriser la construction d'une parole politique de qualité. En montrant que le processus d'ouverture et de détournement des tiers espaces pour y participer politiquement est fragile, nous mettons en avant l'existence d'un pouvoir normatif, capable d'imposer ce que doit être la participation dans ces espaces. Nous montrons effectivement qu'une " police normative » exercée par les communautés de GitHub recadre la participation vers les usages prévus, ce qui limite donc, in fine, la portée politique de ces espaces.
\end{abstract}

Avec le développement des technologies de l'information et de la communication numériques (TICN), la littérature sur la participation s'est largement interrogée sur leur capacité de transformation démocra-

1 Édouard Bouté est ingénieur en Génie Urbain, titulaire d'un master 2 à l'EHESS en Histoire des Sciences, Technologies et Sociétés. Il est actuellement doctorant en SIC à l'Université de Technologie de Compiègne-COSTECH.

2 Clément MABI est maître de conférences en Sciences de l'Information et de la Communication à l'Université de Technologie de Compiègne-COSTECH. Ses recherches portent sur la participation politique en ligne et les usages citoyens du numérique.

Recherches en communication, $\mathrm{n}^{\circ} 52$ - Article publié le 08/09/2021 
tique : les citoyen 'nes ont-ils elles plus de pouvoir d'agir en démocratie grâce à internet ? Un débat persistant s'est installé avec d'un côté, les tenant·es de la «normalisation », représenté es notamment par Michael Margolis et David Resnick (2000), qui défendent l'idée que celles et ceux qui se mobilisent en ligne se mobilisaient déjà hors ligne. Il n'y aurait donc pas véritablement de transformation des rapports de force dans ce nouveau contexte. En face, les tenant $\cdot$ es de la thèse de la « mobilisation » insistent sur la capacité des TICN à mobiliser de nouveaux publics et à renouveler la participation politique, en la rendant plus inclusive (Hirzalla et al., 2011) et de meilleure qualité (Tolbert et al., 2008).

L'avènement de la culture numérique et la démocratisation de l'expression rendue possible par les réseaux sociaux numériques (RSN) a entraîné une diversification des pratiques et des formes possibles de participation, y compris dans des espaces éloignés des dynamiques institutionnelles (Monnoyer-Smith, 2011 ; Cardon, 2019). Cette diversification est à l'origine d'une nouvelle thèse, celle de la " différenciation », qui constate l'éclatement de l'espace public et encourage à analyser finement les arènes d'expression dans toute leur diversité pour correctement saisir la participation qui s'y déroule (Bennett \& Segerberg, 2012 ; Monnoyer-Smith \& Wojcik, 2014). L'un des enseignements majeurs des travaux inscrits dans cette approche est qu'il est souvent difficile de prédire là où les échanges politiques vont effectivement émerger (Jouët \& Le Caroff, 2013). Scott Wright $(2012,2016)$ montre que les espaces d'expression en ligne non destinés à la discussion de contenus politiques peuvent toutefois accueillir de tels échanges. Prolongeant la théorie des « tiers-lieux » (Oldenburg, 1989), le chercheur britannique qualifie ces espaces de «tiers espaces » et explique qu'ils amélioreraient la qualité des échanges et favoriseraient la construction d'une parole politique. Plus récemment cependant, de nouveaux travaux sont venus pointer « le désenchantement de l'internet » et la « brutalisation » du débat public qui limitent la qualité des échanges en ligne (Badouard, 2017).

Dans cet article, nous proposons de prolonger ces débats académiques en interrogeant le pouvoir normatif des tiers espaces, c'està-dire la capacité du dispositif à imposer une lecture de ce que doit être la participation politique dans ces espaces ${ }^{3}$. En effet, notre enquête

3 Le débat normatif qui interroge ce qui relève de la politique dans les espaces en ligne est ancien et couvert par une littérature très fournie. Nous renvoyons ainsi à l'utile synthèse en français de Fabienne Greffet et Stéphanie Wojcik (2008) et aux 
montre que le processus d'ouverture et de détournement des plateformes numériques en tiers espaces d'expression citoyenne est fragile et ne se fait pas sans rencontrer d'opposition de la part des communautés présentes sur la plateforme. Dans certains cas, s'exerce effectivement une « police normative » qui recadre la participation vers les usages prévus, et limite donc, in fine, leur portée politique. Ainsi, la dimension politisée des échanges dans les tiers espaces n'est pas automatique. Qu'est-ce qui rend donc, concrètement, une arène numérique propice à la participation politique?

Pour répondre à notre questionnement, nous mobiliserons les études sur la matérialité des dispositifs qui insistent sur les modèles d'action et le « faire faire » dont le design des TICN est porteur pour organiser la participation (Badouard, 2014 ; Mabi, 2016). Nous ferons dialoguer ces travaux avec la sociologie des usages (Akrich, 1987 ; Jouët, 2000) afin de défendre l'hypothèse qu'une entrée par les dispositifs techniques ne suffit pas pour saisir la manière dont se construit le pouvoir d'agir en ligne, et qu'une réflexion plus large sur la structuration «d'environnements capacitants » doit être menée. Pour analyser la dimension « écologique » du pouvoir d'agir numérique (qui prend en compte l'environnement autour de la technique), nous proposons donc une approche qui croise une attention pour les affordances (ce que la technique invite à faire) et les littératies (les compétences mobilisées pour utiliser les outils). Le parti pris est que pour capter les dynamiques de construction du sens, l'usage doit être analysé en tension avec les propriétés des supports qui le rendent possible. Pour « suivre » ces affordances et les processus qui font advenir les pratiques démocratiques, nous proposons d'associer deux niveaux d'analyse qui permettront d'insister sur la dimension relationnelle et socialement construite des activités démocratiques. Dans un premier temps, nous

travaux de Laurence Monnoyer-Smith (2011) et de Todd Graham, Daniel Jackson et Scott Wright (2016), qui invitent à considérer la démocratie en ligne dans une optique délibérative, basée sur des échanges discursifs qui invitent à l'émergence de visions partagées. Ainsi, les formes de participation politique analysées dans cet article désignent les mobilisations et prises de parole dans l'espace public numérique en dehors des processus du système politique institutionnel, ayant pour objectif de contester ou de critiquer le régime politique en place. Cette définition permet d'interroger l'élargissement des répertoires d'action traditionnels et de comprendre la «parole politique » dans une acception large comme «l'expression d'un jugement politique, quels que soient les arènes et les dispositifs techniques à travers lesquels elle se diffuse » (Monnoyer-Smith, 2011, p.160). 
porterons une attention fine à la configuration technique du dispositif, aux effets de contrainte et aux littératies mobilisées. Dans un second temps, nous nous intéresserons à l'actualisation du modèle dans l'usage.

L'enquête restituée ici part d'un cas particulier, la plateforme de « social coding » GitHub, initialement prévue pour l'échange, le partage, la modification et la discussion de codes informatiques par des communautés de développeur·euses. L'enquête que nous avons menée montre que dans certaines situations, l'usage de GitHub est détourné de sa mission principale (produire collectivement le meilleur code possible) pour servir de support à des échanges plus politisés et militants. La dimension collaborative des usages, ainsi que la présence d'outils permettant de débattre sur le code, semblent effectivement favorables à la construction d'une parole collective qui ne se limite pas qu'à la production de code, faisant ainsi de GitHub une plateforme propice à être considérée comme un tiers espace. Cependant, notre analyse montre également que le dispositif et les communautés qui y sont installées de longue date parviennent en réalité à limiter la réappropriation politisée de la plateforme. Comment expliquer ce décalage ? Un tiers espace peut-il se développer lorsque des communautés de pratiques occupent le dispositif, et que celui-ci distribue de manière différenciée la possibilité de s'exprimer et la légitimité de l'expression?

Nous mobiliserons deux cas d'étude pour appuyer notre réflexion. Le premier concerne le GitHub de Regards Citoyens, une association qui promeut et met en place depuis 2009 des dispositifs numériques de transparence parlementaire en France, à l'image de son site web nosdeputes.fr. Afin de participer elle-même à ce processus de transparence et d'être exemplaire, l'association ouvre les codes informatiques de ses sites web via GitHub ${ }^{4}$.

Le second cas concerne les importantes manifestations qu'a connu la Catalogne en octobre 2019 suite à la condamnation de 13 indépendantistes pour sédition et désobéissance. Lors de ces manifestations, les militant·es catalan'es ont utilisé une application mobile proposée par le mouvement Tsunami Democtràtic permettant de coordonner des rassemblements à des fins de désobéissance civile. Cette application était notamment téléchargeable depuis un dépôt GitHub. Le 23 octobre 2019, la Guardia Civile a sommé GitHub de retirer l'application de sa

4 Pour plus de détails voir Bouté, É. (2018). L'Engagement citoyen en contre-démocratie. Une analyse des dispositifs de surveillance des élu·e $\cdot s$. Mémoire de master 2. EHESS. 
plateforme, ce que fit l'entreprise tout en rendant publique la demande de retrait. En signe de résistance, les militant·es ont retravaillé le texte de demande de retrait directement sur GitHub, comme s'il était un fichier contenant du code informatique.

Cette enquête a été menée en deux temps. D’abord, nous avons réalisé une analyse techno-sémiotique du design de la plateforme GitHub, en insistant sur la dimension collaborative de ce dispositif qui agence des espaces de partage de codes informatiques et des espaces de discussion à propos de ces codes. Puis nous avons consulté l'ensemble des espaces de discussion ayant été ouverts dans les deux cas d'étude. Nous nous sommes alors focalisés sur l'analyse des espaces de discussion où la participation politique a surgi, en observant le déroulé des échanges, en portant attention aux effets de la matérialité des espaces de discussion sur ces échanges, au rôle des participant·es dans l'évolution de la discussion, ainsi qu'aux conséquences de ces échanges. Dans le cas de Regards Citoyens, notre analyse concerne un espace de discussion où 8 messages ont été échangés. Dans le cas catalan, celle-ci concerne 8 espaces de discussion où 95 messages ont été échangés.

\section{GitHub, un dispositif pour modifier le code et discuter sur la technique}

La plateforme GitHub a été lancée sur le web en 2008 par deux entrepreneurs américains, avant d'être rachetée par Microsoft dix ans plus tard pour 7,5 milliards de dollars ${ }^{5}$. Ce qui était à l'origine un projet sans vocation commerciale peut être qualifié de plateforme de social coding, puisqu'elle vise à mettre en relation des développeur·euses afin de favoriser la collaboration et la délibération autour de la construction de logiciels. GitHub met ainsi le code informatique au cœur de la communication entre développeur·euses et rend compte que "le développement informatique est un processus centré sur l'activité humaine », c'est-à-dire qui est avant tout une activité de «négociation du sens » entre différent·es collaborateur·rices qui développent un logiciel (Paz \& Savaroche, 2018). GitHub s'est ainsi imposé comme un outil très utilisé par les communautés du logiciel libre (Schulz, 2019).

Dans cet espace, les informaticien·nes hébergent leurs codes et les partagent à la communauté afin que ceux-ci puissent être complétés,

5 https://www.lemonde.fr/pixels/article/2018/06/04/le-rachat-de-github-par-microsoft-suscite-des-inquietudes-chez-les-developpeurs_5309561_4408996.html 
modifiés, discutés et améliorés. Dans la documentation de la plateforme, il est expliqué que " la révision ${ }^{6}$ du code est le chemin le plus sûr vers un meilleur code $»$. Ce projet est ainsi imprégné de la vision libérale (dite "Open Source ») du logiciel libre, qui soutient que l'ouverture des codes est à la base de meilleures performances techniques et donc de la fabrication de logiciels de meilleure qualité, à l'inverse d'une vision plus libertaire (dite «Free Software ») qui se défait de l'idéologie de la performance pour défendre avant tout le partage et la reprise des codes d'autrui comme symbole de la liberté des individus (Gleizes, 2000 ; Broca, 2013 ; Loveluck, 2015). Cette vision libérale de la culture numérique du partage est « mise en technologie » (Badouard, 2014), traduite dans le script de la plateforme, par le biais de différentes fonctionnalités organisant la collaboration entre développeur euses sur GitHub. La première étape est la publication du code sur la plateforme. Pour ce faire, un·e utilisateur·rice crée un "repository », à savoir un " dépôt » qui «contient tous les fichiers d'un projet, y compris l'historique des révisions $^{8} \gg($ voir Figure 1).

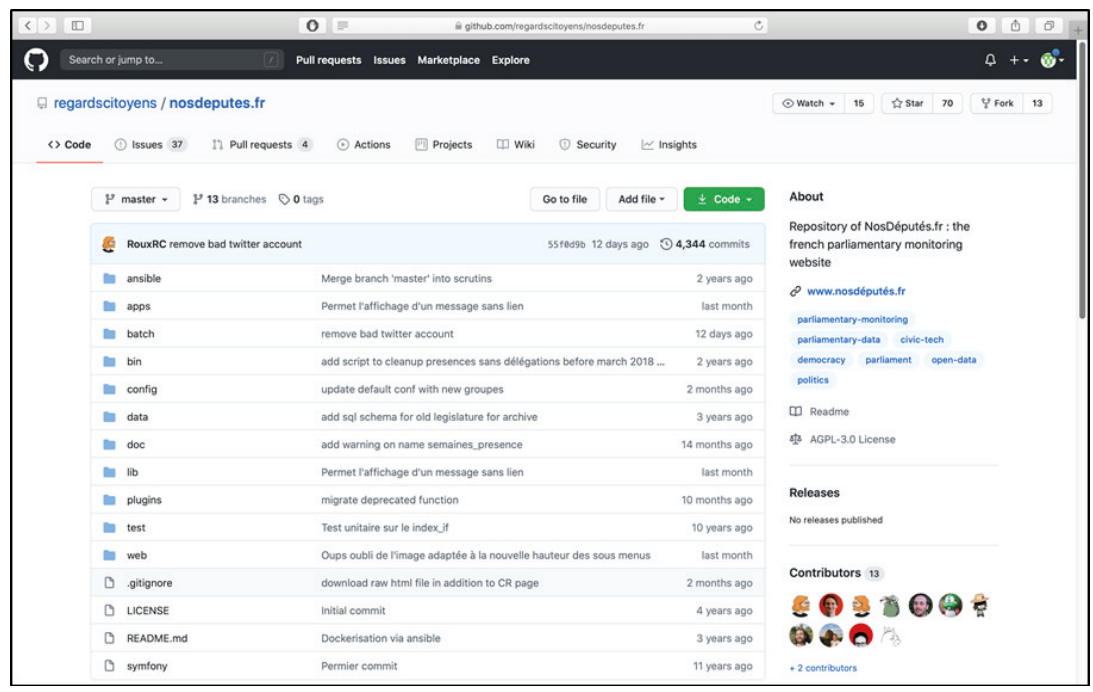

Figure 1. Exemple $d u$ repository $d u$ projet nosdeputes.fr de l'association Regards Citoyens

6 En informatique, une révision est synonyme de « version » d'un fichier de code.

7 Notre traduction, https://github.com/features

8 Notre traduction, https://github.com/new 
Jusqu'en janvier 2019, le caractère public des projets - visibles de tou tes, duplicables et modifiables par les membres de la plateforme était la norme, dans le sens où les comptes gratuits (majoritairement utilisés) n'avaient pas la possibilité de rendre privés leurs projets. Cette transparence est à la base du social coding, dans la mesure où elle favorise le travail collectif autour du code (Thung et al., 2013) et permet de "déduire les objectifs techniques et la vision de quelqu'un d'autre lorsque du code est édité ", ce qui a pour conséquence d'améliorer la qualité de la collaboration (Dabbish et al., 2012). Dans les dépôts, seul·es les membres autorisé es par les propriétaires des projets peuvent directement modifier les fichiers de code sans en demander la permission. Lorsque des membres extérieur'es à un projet ne disposent pas des droits d'écriture nécessaires, mais souhaitent toutefois s'y impliquer, la plateforme met à disposition quatre actions permettant de collaborer autour du code entre membres et non-membres d'un projet : le fork, le commit, la pull request et l'issue. Ces quatre actions permettent deux modes de participation : la participation modificative - où l'on participe en modifiant le code - et la participation discursive - où l'on participe en discutant le projet, le code tel qu'il est, ou encore le code modifié (voir Figure 2).

La première action est le fork. Il est à la base de toute participation modificative et correspond à la duplication d'un projet public d'un $e$ autre développeur-euse vers son propre compte pour en créer une nouvelle version. Cette fonctionnalité collaborative s'inscrit dans le prolongement de la culture libertaire du logiciel libre dans la mesure où, si le projet avance sur la base d'un consensus minimal, une nouvelle trajectoire peut immédiatement être proposée en cas de désaccord sans entraver le projet de départ. À l'intérieur d'un projet ainsi dupliqué, les développeur euses peuvent intervenir en proposant des modifications par un " commit », que Stéphane Couture définit comme " un acte informatique qui consiste à valider formellement une modification du code source d'un logiciel ». Pour le dire autrement, "le commit consiste à publier les changements apportés au code source, pour les rendre accessibles aux autres acteurs impliqués dans le projet, voire même, dans le cas de logiciels libres, au public en général » (Couture, 2012). Afin d'intégrer les modifications réalisées au projet d'origine, il faut en faire la demande aux propriétaires du projet par le biais d'une pull request $(\mathrm{PR})$. GitHub définit cela comme une action permettant 
"d'informer les autres des modifications apportées ${ }^{9}$ ». Dans sa documentation, GitHub explique qu'un «meilleur code commence avec une $P R$, une conversation vivante à propos des changements où l'on peut échanger des idées, assigner des tâches, discuter des détails, et effectuer des révisions ${ }^{10} »$. Concrètement, lors d'une PR, le code originel et le code modifié sont mis en comparaison ligne par ligne afin d'aider à l'examen des changements proposés. Dans le même temps, un espace de discussion est activé dans le dépôt qui contient le projet d'origine, afin de décider de l'intégration ou non de la modification proposée pour le projet. Dans cet espace de débat, les participant·es doivent justifier et expliquer leurs choix pour démontrer la pertinence de leur modification pour l'amélioration du projet auprès de leurs propriétaires. Des participant es n'ayant pas écrit directement le code peuvent venir contribuer aux échanges, en posant des questions ou encore en exprimant des remarques qui peuvent potentiellement sortir du champ purement technique. Enfin, la fonctionnalité des issues permet elle aussi d'ouvrir un espace de discussion. Cependant, à la différence de la PR, le lancement du débat n'est pas lié à une modification du code. Une issue permet ainsi à des personnes n'ayant pas forcément de compétences dans l'écriture du code informatique de s'impliquer en faisant une remarque ou en alertant sur un bug repéré, etc. De plus, contrairement à la PR, la fonctionnalité des issues peut être désactivée sur un dépôt.

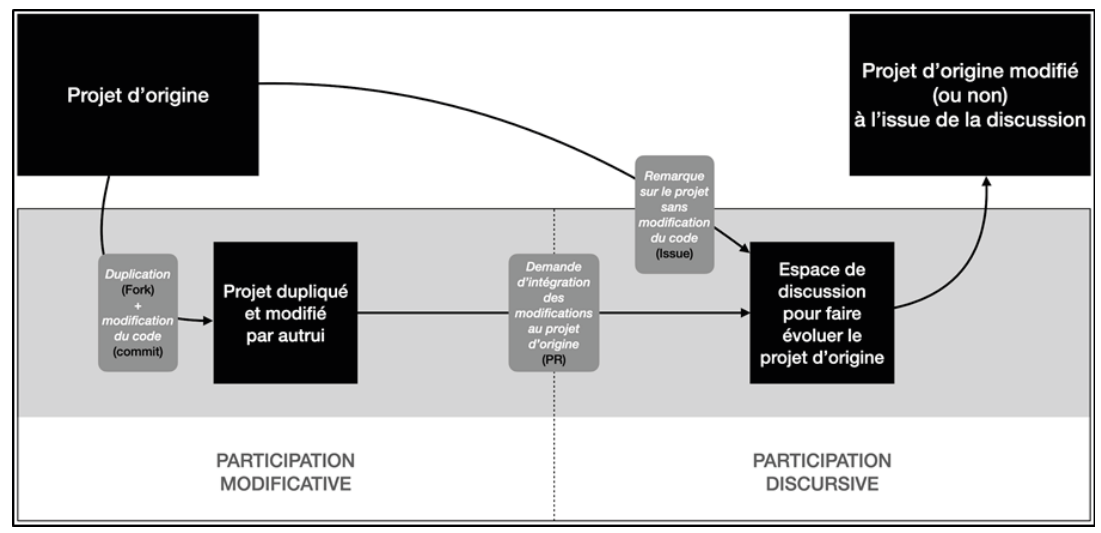

Figure 2. Processus d'écriture collaborative du code sur Github

9 https://docs.github.com/en/github/collaborating-with-issues-and-pull-requests/ about-pull-requests

$10 \mathrm{https}$ //github.com/features 
Un tel espace de discussion sur la technique crée une dynamique dialogique entre les utilisateur-rices. Dans le cas d'une PR, l'espace de débat généré par cette action est constitué de quatre onglets (voir Figure 3). Deux rendent compte des participations modificatives : l'un présente les fichiers concernés par la discussion (onglet « Commits »). Le second, "Files changed », rassemble ceux qui ont été modifiés et rend visible les lignes de code modifiées. Les lignes supprimées sont affichées en rouge et les lignes ajoutées sont de couleur verte pour faciliter la lecture. Un troisième onglet « checks » donne le résultat du contrôle de qualité du code modifié. Les différents points de contrôle sont définis en amont et doivent tous être validés par la modification proposée. La mise en place de ce contrôle de qualité est optionnelle. Par exemple, pour le GitHub de Facebook, les membres qui proposent des modifications doivent signer un document « Contributor License Agreement », et une batterie de tests doivent être passés avec succès avant intégration du code au projet d'origine. L'effectivité de ces différents éléments est indiquée dans cet onglet.

Enfin, l'onglet principal, "Conversation », qui s'affiche lorsque l'on ouvre une PR, est celui de la discussion elle-même. Cependant, les différents messages de la discussion ne sont pas les seuls éléments présents dans cet onglet, puisque dès qu'une modification technique est réalisée, ou qu'une action est effectuée, celle-ci est notifiée dans le fil de la discussion. Par exemple, si une personne souhaite approuver un changement, elle peut le notifier et son approbation apparaitra dans le fil de la discussion à côté d'une coche. Si un fichier de code est modifié en lien avec la discussion, cela est également notifié. Enfin, à la fin de la discussion, si la modification est validée ou refusée, cela est indiqué. On peut ainsi retracer l'ensemble des échanges et des modifications que ceux-ci ont engendré à la lecture de cet espace de discussion. De plus, il est possible d'exprimer son approbation ou sa désapprobation en ajoutant un emoji aux messages des autres. La qualité des participant es est également indiquée à chacun des messages postés : propriétaire ou membre (dans le cas où le projet est celui d'une organisation, la qualité de « membre » indique que l'internaute fait partie de cette organisation), auteur trice (personne à l'origine de la PR), contributeur trice (personne qui a déjà contribué sur le projet). Dans le cas d'une première contribution à une discussion, rien n'est affiché. 


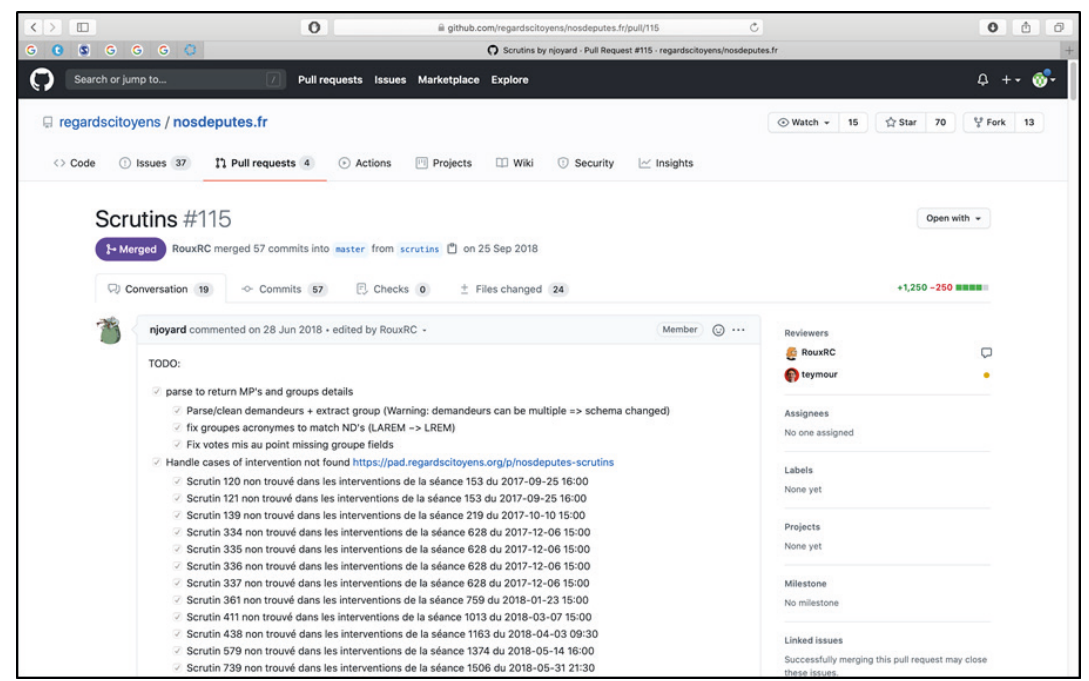

Figure 3. Exemple de Pull Request, ouverte sur l'onglet "Conversation »

Cette distribution des rôles est importante pour identifier le pouvoir d'agir de l'utilisateur·rice sur le projet. En effet, seul·es les propriétaires/membres du projet concerné peuvent décider d'accepter ou non les modifications du code mises en discussion. À l'image de projets collaboratifs comme Wikipédia, tou·tes les acteur·rices n’ont pas le même poids ni la même autorité sur la production (Cardon \& Levrel, 2009). En conséquence, pour voir leurs propositions de modification acceptées, les participant·es déploient des stratégies discursives qui visent à défendre la légitimité technique de leur proposition. Les discussions initiées visent à enrichir la lecture des lignes de code proposées pour faciliter leur intégration.

Par exemple, sur le GitHub de la plateforme de participation citoyenne Decidim ${ }^{11}$, il est explicitement demandé à ce que les PR répondent à un format particulier pour pouvoir être prises en compte. Le champ dans lequel est rédigé le texte associé à une proposition de modification est pré-rempli avec un certain nombre de consignes pour les auteurs : bien expliquer ce qui est modifié et pourquoi, détailler les issues associées s'il y en a, expliciter si l'on a bien effectué un certain nombre de sous-tâches lors de la modification, ou encore ajouter des captures d'écran pour rendre plus compréhensible la modification. Afin

$11 \mathrm{https}: / /$ decidim.org/ 
de faire intégrer leurs propositions, les utilisateur·rices sont obligé·es de se conformer à ce format de participation attendu (voir Figure 4).

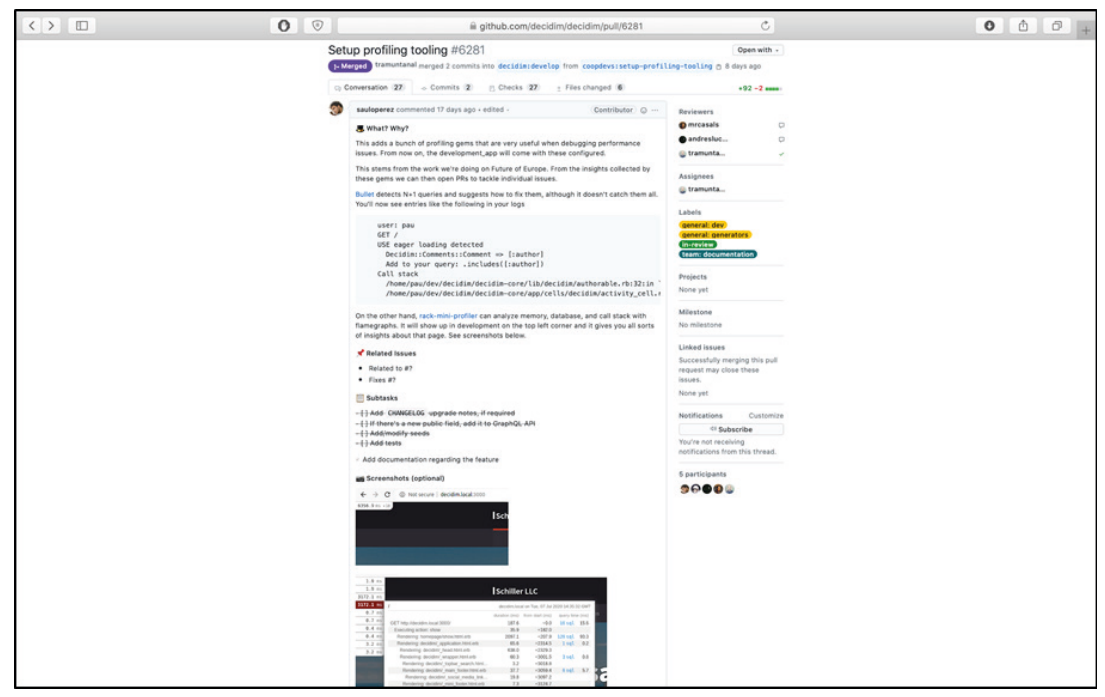

Figure 4. Texte associé au code pour rendre légitime sa participation modifcative auprès des propriétaires d'un projet

\section{GitHub comme espace de participation politique}

Sur GitHub, une certaine manière de participer est proposée, articulant des moments de modification et de discussion du code, et attribuant une place particulière aux participant·es. On parle alors du « script» des technologies pour qualifier la volonté des concepteur·rices à « prédéterminer » les manières dont les utilisateur·rices sont invité·es à se saisir du dispositif technique (Akrich, 1987). Ainsi, sur internet, pour Lawrence Lessig (2000), « le code fait loi » (« code is law »), c'està-dire que l'architecture de l'espace numérique régit nos possibilités d'action dans cet espace, et définit en conséquence des hiérarchies entre acteur $\cdot$ rices.

Or, les communautés qui investissent depuis longtemps et majoritairement cette plateforme open source adhèrent à son script et aux principes qui président aux modes de participer. Si les communautés du logiciel libre ont certes des règles de fonctionnement plutôt souples et informelles (Demazière et al., 2011), elles n'en ont pas moins des valeurs structurantes qui rendent légitimes certaines participations plus que d'autres. Comme l'explique Anne Bellon (2014), « l'action 
collective s'appuie avant tout sur des pratiques collectives fortement normées 》 où les compétences techniques et le partage de principes éthiques sont à la base de la cohésion communautaire et sont une condition à la légitimité de l'expression. Cependant, le modèle de participation libéral inscrit dans le script de GitHub est parfois mis à l'épreuve par des utilisateur-rices qui utilisent la plateforme dans une optique différente, plus critique. En participant à partir du code (en modifiant les fichiers hébergés sur GitHub) et autour du code (en discutant ces modifications), des internautes font valoir leur opinion et produisent des formes de participation politique, contribuant à détourner le script de GitHub.

\subsection{Regards citoyens : la collaboration critique}

L'association Regards Citoyens milite pour l'amélioration de la transparence des institutions démocratiques, afin que celles-ci soient mieux comprises par les citoyen'nes. Elle est ainsi à l'origine de plusieurs plateformes numériques, dont nosdeputes.fr qui récolte et présente de façon accessible différentes informations déjà publiques sur l'activité parlementaire. Dans un souci de symétrie, l'association organise également la transparence sur ses activités. Elle publie donc l'ensemble des codes sources de ses projets dans des dépôts GitHub :

« Le collectif Regards Citoyens a comme objectif de concevoir des outils permettant aux citoyens de mieux comprendre la démocratie. Afin de mieux évaluer la qualité de nos outils et de permettre à chacun d'en créer de nouveaux, nous publions l'ensemble des logiciels et données de nos projets en téléchargement sous licences libres ${ }^{12}$.»

En rendant ainsi transparent l'ensemble de ses projets sur GitHub, l'association ouvre son travail à la critique citoyen'ne. Lors de notre enquête, nous avons observé les discussions autour d'une proposition citoyenne de modification du code qui souhaite modifier la présentation des indicateurs sur l'activité des parlementaires pour éviter d'encourager « l'antiparlementarisme ${ }^{13}$ ». Effectivement, nosdeputes.fr propose, entre autres, une représentation graphique de l'activité des député·es :

12 https://www.regardscitoyens.org/publication/

$13 \mathrm{https}: /$ github.com/regardscitoyens/nosdeputes.fr/pull/68 
présence en hémicycle, nombre de prises de parole, de questions orales posées au gouvernement, etc. Des captures d'écran sont régulièrement réalisées par des internautes qui les mobilisent pour saluer ou dénoncer l'investissement d'un·e député·e au cours de son mandat d'élu·e (voir Figure 5).

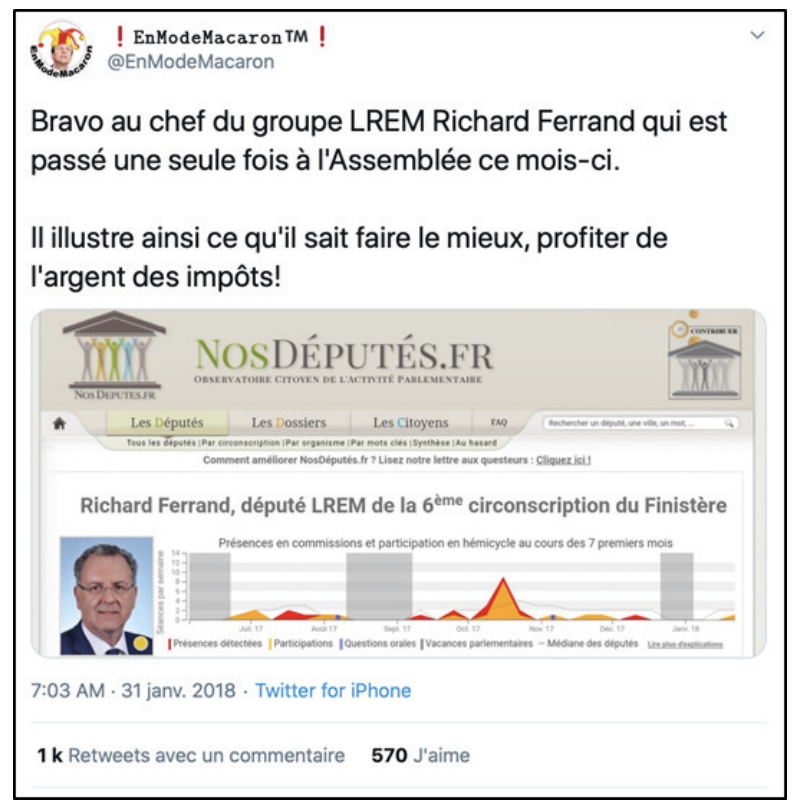

Figure 5. Capture d'écran du graphique représentant l'activité des parlementaires sur nosdeputes.fr, mobilisée dans un tweet

Or, pour un internaute venu proposer une modification, l'indicateur de " présences détectées » est problématique, dans la mesure où il ne se base pas sur une information publiée par l'institution, mais fait l'objet d'une reconstruction par Regards Citoyens. Effectivement, pour quantifier la "présence » des élu·es à l'Assemblée nationale, l'association prend pour convention le fait qu'une prise de parole au sein de l'hémicycle équivaut à une présence en hémicycle. Pour l'internaute à l'origine de la proposition, ce fonctionnement est biaisé car il invisibilise les député es présent es qui ne prennent pas la parole. Il note également que ce biais n'est pas explicité sur le site, notamment dans la légende des graphiques, induisant potentiellement une lecture faussée de l'activité des parlementaires. À travers une participation modificative, il propose donc de détailler la légende et rendre visible les choix de l'association (voir Figures 6 et 7). 


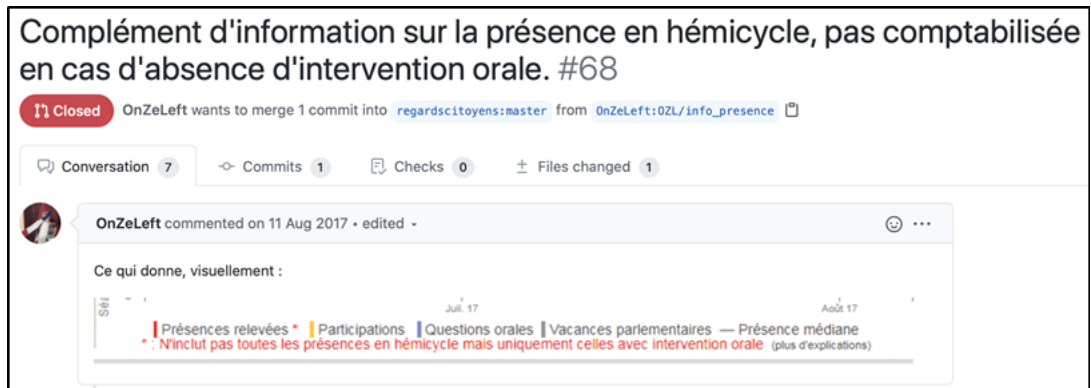

Figure 6. Rendu graphique de la participation modificative de l'internaute

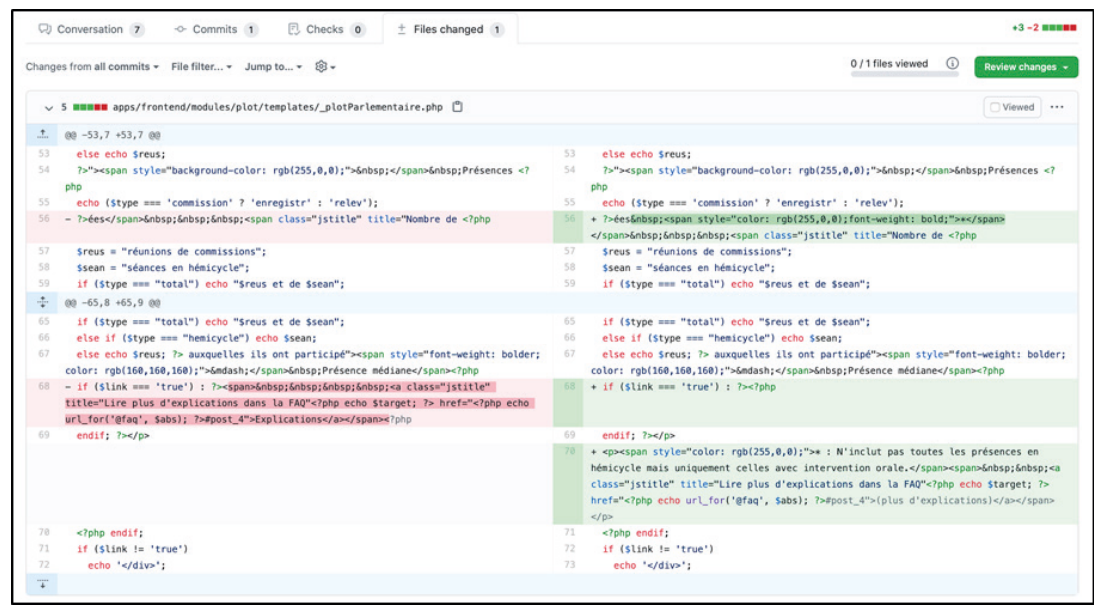

Figure 7. Modifications du code par l'internaute

Un échange de 8 messages a ensuite lieu dans l'espace de discussion ouvert lors de la PR. Dans un premier temps, l'internaute ne justifie pas directement ce qui a motivé sa participation modificative. Un membre de l'association lui fait alors plusieurs remarques concernant sa proposition de modification. Ses remarques sont à la fois d'ordre technique (concernant la couleur, la mise en page, l'esthétique) et d'ordre réflexif (est-ce à l'association de lutter contre les mauvaises interprétations ?). Cet ensemble de remarques pousse donc l'internaute à expliciter les raisons de sa participation dans un deuxième temps. Il justifie alors sa proposition en affirmant qu'il est clair que l'indicateur actuel occasionne du «bashing anti-parlementaire ». Il explique que «Fermer les yeux sur [cela] paraît démocratiquement dangereux. 
L'association doit avoir conscience de l'importance et l'écho que ce site a pris et donc de la responsabilité qui va avec ». Il justifie ensuite ses choix techniques, mais admet également que la modification, telle que proposée, «n'est d'ailleurs pas idéale » et que le problème pointé ne saurait être vraiment résolu par cette seule modification.

Malgré sa justification, la PR est refusée par les membres de l'association qui estiment que les modifications ne peuvent pas être intégrées telles quelles. Cependant, il est admis que l'indicateur de présence peut générer de la mécompréhension. Les membres prolongent donc la discussion afin de réfléchir à une solution alternative pour résoudre le problème. À la suite de cet échange où l'auteur de la PR ne participe plus, il est finalement décidé de modifier le nom de l'indicateur « présences détectées » par « présences relevées ». Un nouvel indicateur sera également ajouté à la légende pour expliciter que la présence en hémicycle est une information non publique.

Cet échange rend compte des discussions politiques qui se jouent autour des choix de code informatique. Ce type de participation contribue à une citoyenneté active, alerte et technicienne où la participation passe par la contribution à l'amélioration de dispositifs numériques en raison de leur performance politique. Dans la PR analysée, cette participation politique, en raison de sa forme, est donc valorisée par les membres de l'association : "merci de venir participer à ce débat d'une manière constructive, personnellement j'apprécie l'approche Pull Request sur GitHub»; "merci de cette initiative, j'apprécie aussi cette approche, plus propice au dialogue que les salves de 140 caractères ».

\subsection{Tsunami Democràtic : la dénonciation militante}

Le second cas d'étude est lié aux importantes manifestations que la Catalogne a connu en octobre 2019 suite à la condamnation de 9 indépendantistes pour sédition et désobéissance ${ }^{14}$. Lors de ces manifestations, les militant · es catalan·es ont utilisé une application mobile proposée par le mouvement Tsunami Democràtic permettant de coordonner des actions de désobéissance civile. Cette application était notamment téléchargeable depuis différents dépôts GitHub où elle était hébergée. Le 23, puis le 26 octobre 2019, le gouvernement espagnol a sommé l'entreprise de retirer l'application de sa plateforme, ce qu'elle fit tout

$14 \mathrm{https}$ ://www.lemonde.fr/international/article/2019/10/14/neuf-dirigeants-catalanscondamnes-a-des-peines-de-prison_6015398_3210.html 
en rendant publique la demande de censure des dépôts concernés. Effectivement, lorsqu'un État fait parvenir à GitHub une demande de suppression d'un contenu ayant été déclaré illégal dans leur juridiction locale, le texte de la demande est rendu public dans un dépôt public appartenant à GitHub appelé « gov-takedowns ${ }^{15}$ » (voir Figure 8). Cela signifie que si les fichiers hébergés dans cet espace ne sont pas à proprement parler des fichiers contenant du code, ils sont traités comme tel par la plateforme, et le même type d'actions peut être effectué (fork du dépôt « gov-takedowns », commit, PR, etc.).

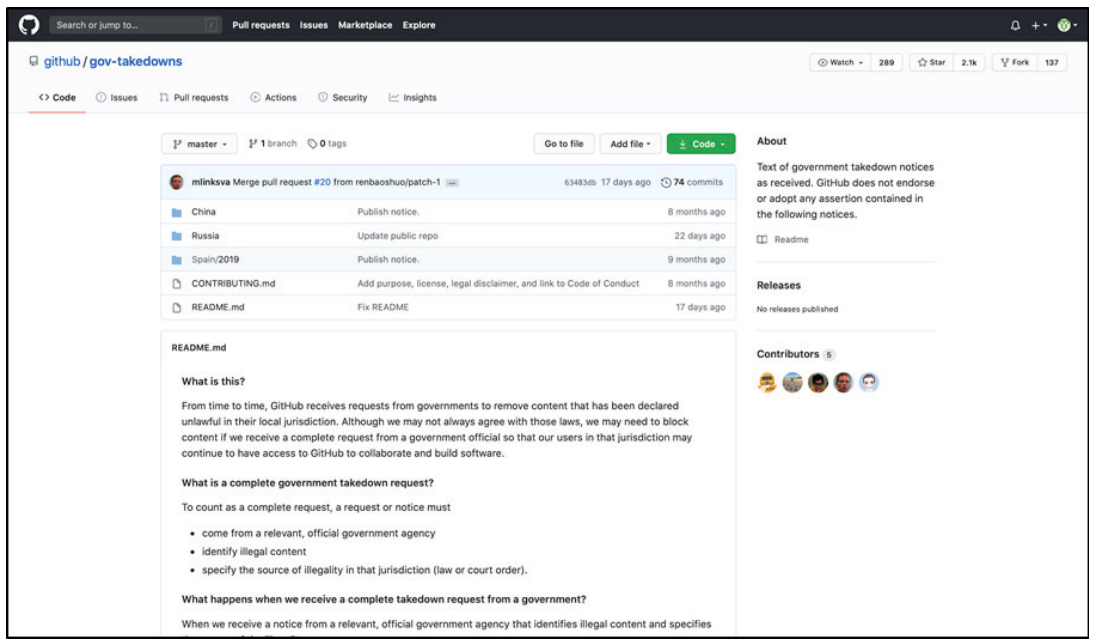

Figure 8. Dépôt « gov-takedowns » appartenant à Github

Ces deux demandes de suppression des dépôts rendues publiques expliquent que "l'Espagne subit actuellement une série d'émeutes». Étant en lien avec ces «émeutes 》, le mouvement Tsunami Democràtic est désigné comme étant une « organisation criminelle poussant des individus à commettre des attaques terroristes ». Il est par ailleurs expliqué que l'application téléchargeable depuis GitHub permettrait de « coordonner les émeutes et actions terroristes » et devrait donc être interdite en conséquence.

Cette décision a suscité de vives réactions au sein des communautés évoluant sur GitHub. Dans un premier temps, 8 internautes proindépendances se sont mobilisé·es pour modifier, comme s'il s'agissait

15 https://github.com/github/gov-takedowns/ 
d'un fichier contenant du code, le texte de la demande du 23 octobre. Le dépôt " gov-takedowns » a ainsi été forké, des commits ont été réalisés, et 8 PR ont été ouvertes. Deux internautes ont par exemple supprimé l'ensemble du texte pour écrire à la place une invitation à aller télécharger l'application de Tsunami Democràtic via Telegram ${ }^{16}$ (voir Figure 9). D'autres modifications expriment plus explicitement des revendications. Deux d'entre elles portent par exemple les titres de « make Spain an actual democracy ${ }^{17}$ » et «Dear GitHub, you've been played by a failed state $^{18} \gg$ (voir Figure 10). Dans une autre proposition de modification, un e internaute a publié un texte regrettant que l'Espagne trouve une place dans ce dépôt aux côtés de la Chine et de la Russie. L'attitude du gouvernement espagnol y est vivement critiquée et des liens URL renvoyant vers des articles de presse ou encore vers des billets de blog publiés par l'ONG Amnesty International sont partagés ${ }^{19}$. Ces différents exemples témoignent de la dimension revendicative de ces participations modificatives. Le détournement des fonctionnalités de la plateforme est radical : il n'y a pas de volonté de collaborer avec les propriétaires du dépôt comme dans le précédent cas d'étude. Il s'agit au contraire de dénoncer l'action de GitHub comme celle du gouvernement espagnol.

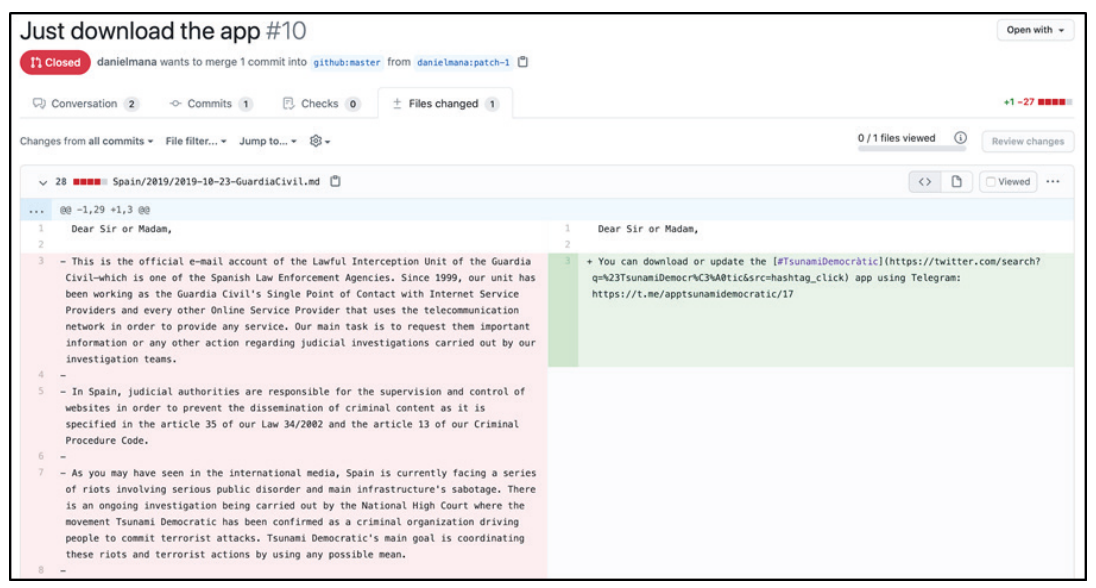

Figure 9. Comparaison du fichier original avec sa modification invitant à télécharger l'application via Telegram

$16 \mathrm{https} / /$ github.com/github/gov-takedowns/pull/10

$17 \mathrm{https} / /$ github.com/github/gov-takedowns/pull/8

$18 \mathrm{https} / /$ github.com/github/gov-takedowns/pull/9

$19 \mathrm{https} / /$ github.com/github/gov-takedowns/pull/11 


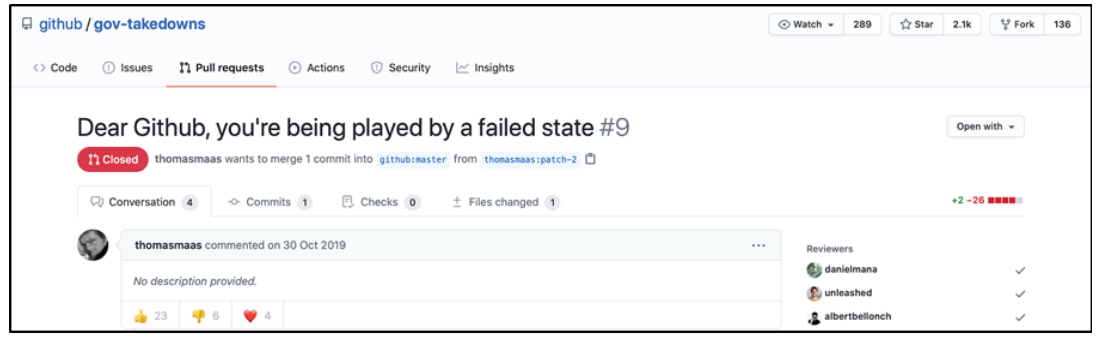

Figure 10. Proposition de modification au titre revendicatif

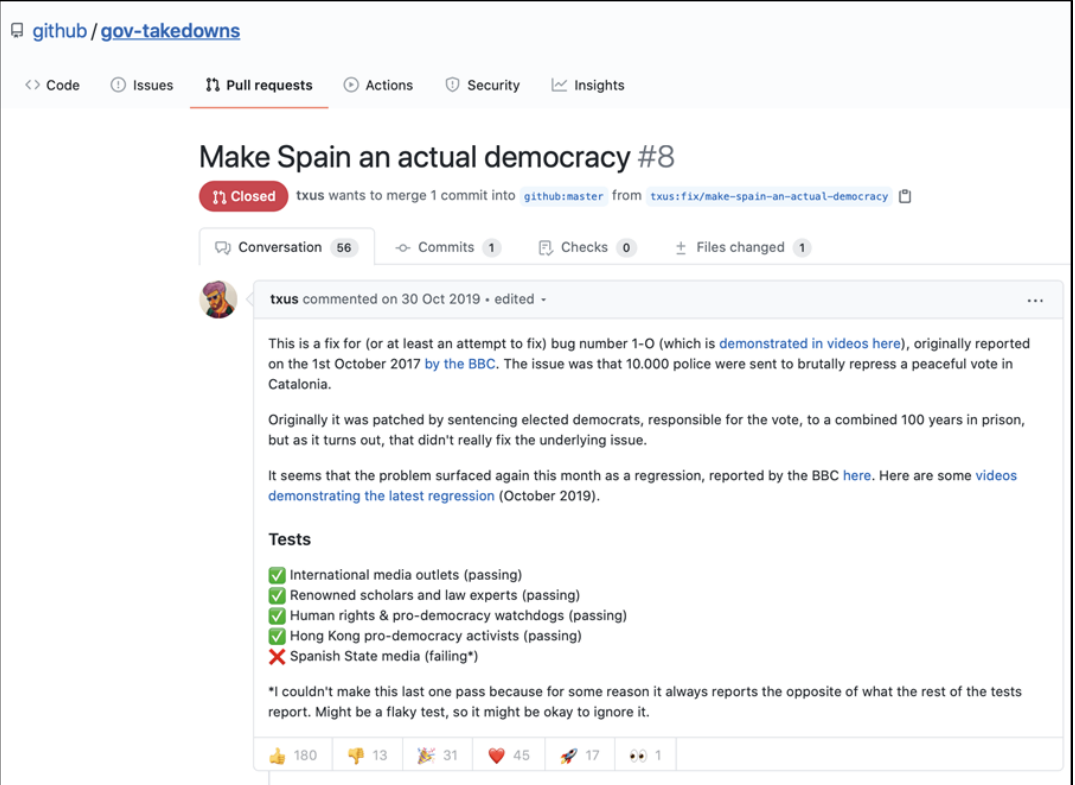

Figure 11. Espace de discussion d'une des PR dénonciatrices de la situation en Espagne

Dans les espaces de discussion ouverts par ces propositions de modification, la participation politique s'est poursuivie et intensifiée : 87 commentaires ont été publiés dans l'ensemble des 8 espaces de discussion. Dans la PR ayant généré plus de la moitié des commentaires (56), les échanges jouent sur les codes habituels des formes de la discussion sur GitHub ${ }^{20}$. Le membre ayant proposé la modification mobilise des termes courants chez les développeur·euses : "This is a fix for », " the

20 https:/github.com/github/gov-takedowns/pull/8 
issue was 》, «it was patched», etc. (nous soulignons). Dans ce message, il partage des liens hypertextes renvoyant vers différentes vidéos qui rendent compte de la répression subie par les militant·es catalan·es (voir Figure 11). Les réponses à ce premier message reprennent également les termes propres au milieu informatique pour parler du conflit entre le gouvernement espagnol et les indépendantistes catalan·es, comme par exemple ce premier message : "I've also been trying to (unsuccessfully) fix this for a long time. Can I suggest adding this to the Spanish State media test cases? ». Cependant, les messages générés ne sont pas seulement publiés par les militant es indépandantistes, mais aussi par leurs opposant-es qui viennent expliquer que la Catalogne serait au contraire plus totalitaire que l'Espagne, que le contenu du texte de l'auteur est malhonnête, ou encore que le gouvernement doit être plus ferme avec ces militant·es, en faisant par exemple appliquer correctement « l'article $155 »$ qui permet de contrôler les communautés autonomes du territoire espagnol, ou encore en suspendant le gouvernement catalan (voir Figure 12).

De la participation modificative émerge donc un débat polarisé au cours duquel les participant·es montent en généralité dans la discussion, dépassant le simple cadre de la critique de la suppression de l'application sur la plateforme, pour débattre de la légitimité de l'indépendance de la Catalogne.

L'acceptation de GitHub de supprimer l'application suscite elle aussi de vives réactions. Elle est critiquée par les uns : "Thanks to GitHub, people who are in Spain cannot visit a website about a grassroots democratic movement that has hurt nobody ». Mais est aussi saluée par les autres : "What is doing GitHub is great! It is really nice to see a company doing real transparency $\gg$. La participation politique des militant·es catalan·es est dévalorisée par les propriétaires du dépôt et par une partie de la communauté de GitHub. Au final, les modifications proposées sont toutes refusées par deux employé es de GitHub. Cette décision est motivée par le fait que le but affiché de ces participations n'était pas de collaborer au projet, mais d'exercer une dénonciation critique à l'encontre de GitHub et du gouvernement espagnol. L'entreprise ne revient pas sur le fait que les dépôts contenant l'application aient été supprimés et se limite à expliquer que si les PR ne sont pas acceptées, c'est parce que les fichiers publiés sont des verbatim, c'està-dire des fichiers qui reprennent mot pour mot les demandes effectuées par les gouvernements. 


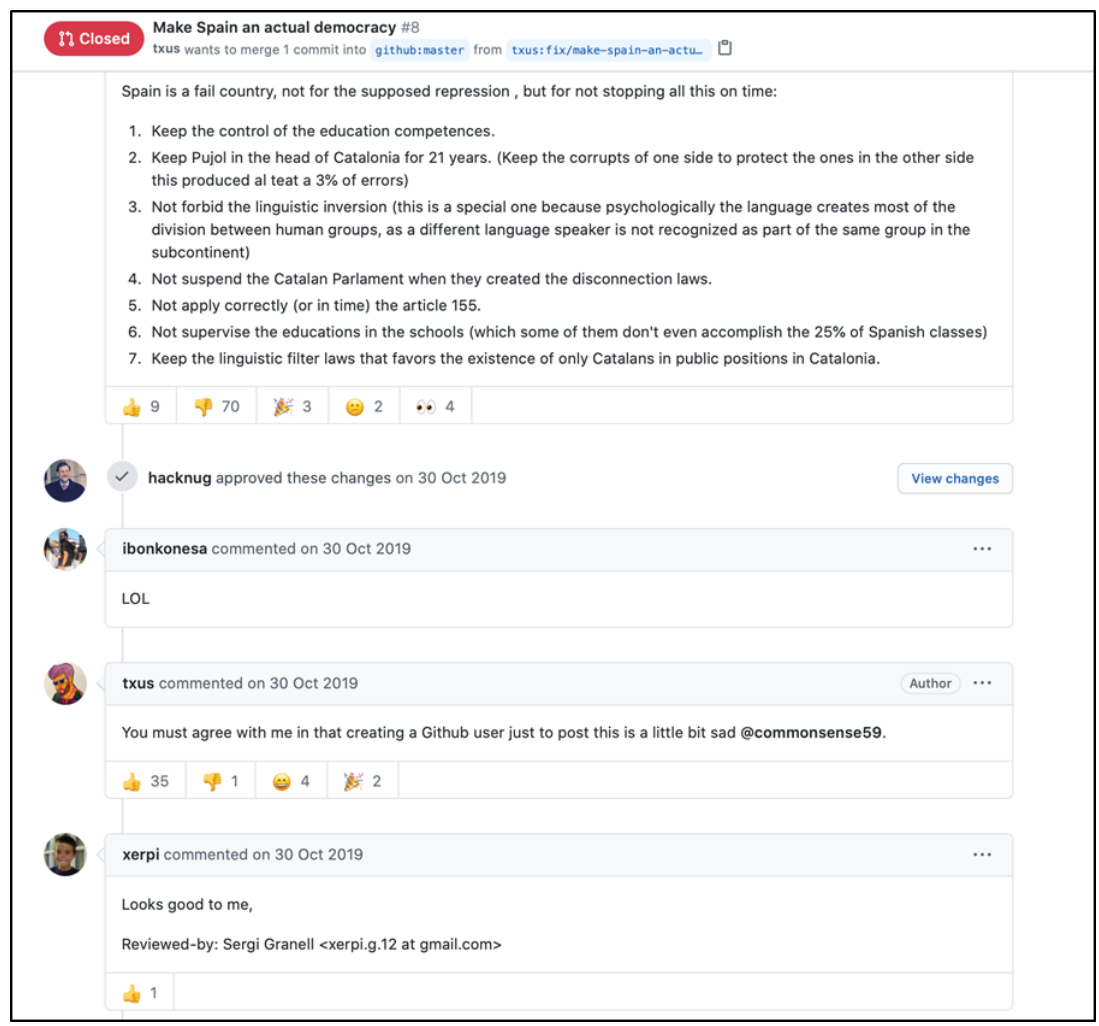

Figure 12. Un des échanges au sein de l'espace de discussion ouvert

\section{Le poids normatif du script plus fort que la politique ?}

Sous certaines conditions, GitHub peut donc être le support d'une participation politique, dans la mesure où la dynamique collaborative pour produire du code informatique permet aux participant es d'exprimer un point de vue, de défendre une cause ou d'échanger avec d'autres membres de la communauté GitHub. Cependant, notre enquête montre que cette participation politique reste fragile dans la mesure où certain·es participant·es exercent une «police normative » afin de veiller à ce que le script d'usage de la plateforme ne soit pas détourné. Parler de « police normative » permet d'abord de mettre l'accent sur le fait que les ressources communicationnelles incorporées dans le script de GitHub distribuent des rôles entre les participant·es qui disposent d'un pouvoir d'agir sur le code différencié, ce qui permet à certain·es 
acteur·rices - notamment les propriétaires des dépôts - d'engager une bataille de sens sur ce que devrait être la participation sur la plateforme. Cela permet également de qualifier la mobilisation dans la discussion de la part de la communauté de développeurs et de développeuses de GitHub qui, en s'exprimant, s'engage également dans cette bataille de sens en défendant l'idée que certaines modalités de participation sont plus légitimes que d'autres. En acceptant (ou non) les modifications proposées, en laissant (plus ou moins) la discussion s'engager, des acteurs dominants exercent à travers des stratégies discursives et techniques un pouvoir de contrôle normatif pour s'assurer que le script d'usage originel de la plateforme n'est pas détourné. Notre enquête nous a permis d'identifier plusieurs manifestations de cette police normative.

La première police, que l'on peut qualifier de « police dialogique » est exercée par les participant·es impliqué·es dans le dépôt, voire par la communauté GitHub de façon plus générale. Dans le cas de Regards Citoyens, on observe que dans un premier temps les critiques se sont inquiétées de la dimension partisane de la proposition de modification relative à l'indicateur de présence des parlementaires, et ce en raison $\mathrm{du}$ profil du porteur de cette proposition de modification. Pour les membres de l'association, l'affichage «\#TeamMacron » de ce participant fait craindre une lecture partisane du code, contraire aux valeurs de l'association :

«Il faut que 1'association fasse attention eu égard de ses statuts, notamment son article 2 et l'obligation de transpartisanité. L'auteur du commit est un militant explicite d'un parti d'après son compte Twitter : https://twitter.com/ onzeleft. Sa description dit «\#TeamMacron \#LREM». Son compte github a visiblement été créé uniquement pour l'occasion. Il s'agit par ailleurs de sa première PR dans nosdeputes. Il peut facilement être envisagé que son but est de modifier nosdéputés de façon partisane [...] L'association doit se protéger de toute attaque sur la présence de modification partisane ${ }^{21}$. ’”

Grâce à la discussion qui suit sa PR, l'auteur de la requête a pu se défendre et expliciter le sens de sa contribution :

21 https://github.com/regardscitoyens/nosdeputes.fr/pull/68 
« Je pense avoir mis en forme la PR de façon totalement non partisane $[\ldots]$ je pense qu'on peut contribuer à ce type de projets en mettant de côté ses engagements. Je n'ai en effet pas caché le mien (j'aurais pu choisir un autre pseudo...) et c'est effectivement ma première contribution à ce projet [...]. Mais je comprends que pour éviter toute attaque, ma contribution soit reprise par d'autres personnes de l'association. Aucun problème avec ça (je souhaitais surtout initier une amélioration et/ou discussion). »

Dans cet échange, l'utilisateur désamorce la dimension politique de sa contribution sur GitHub et rappelle sa volonté de contribuer au projet général en cherchant l'appui de la communauté. De fait, le doute semble levé car la suite de la discussion se concentre sur les modifications à apporter au site web de l'association. Le poids normatif du script - et la place prépondérante que celui-ci accorde aux propriétaires dans la discussion - semble avoir été suffisamment important pour recadrer le participant et dépolitiser sa contribution de manière à ce qu'elle soit acceptée.

Dans le cas catalan, où GitHub a réagi en acceptant le retrait demandé par le gouvernement espagnol, les échanges ont largement porté sur la nature des participations au regard de « l'esprit» de la plateforme, à ce que l'on est en droit d'attendre dans cet espace (voir Figure 13). Le caractère expressément militant des modifications est directement critiqué : " please do not include your political opinions on this document ${ }^{22}$ "; " don't make this repository a personal weapon to your political speech ${ }^{23} \gg$. Plusieurs informaticien nes de la communauté qui participent aux discussions estiment ainsi que GitHub n'est pas le lieu pour ce type de propos : "it's not a platform for discussions $^{24} »$, « Such PRs are obviously out of scope $e^{25} »$. L'objectif de transparence du dépôt est également un argument mobilisé pour critiquer le fait d'avoir proposé une PR : " GitHub transparency repository is not your propaganda ${ }^{26} », ~ « C l o s e$. This repository is nothing to do with pol-

22 https:/github.com/github/gov-takedowns/pull/6. Les échanges sont présentés dans la langue d'origine.

23 Ibid.

24 https://github.com/github/gov-takedowns/pull/11

25 Ibid.

$26 \mathrm{https} / / /$ github.com/github/gov-takedowns/pull/6 
itics or taking a side. It's for transparency of GitHub ${ }^{27} \gg$. En rappelant la mission première de la plateforme, et en militant pour que celle-ci ne soit pas débordée, certain·es utilisateur·rices imposent donc une lecture particulière de ce que doit être GitHub.

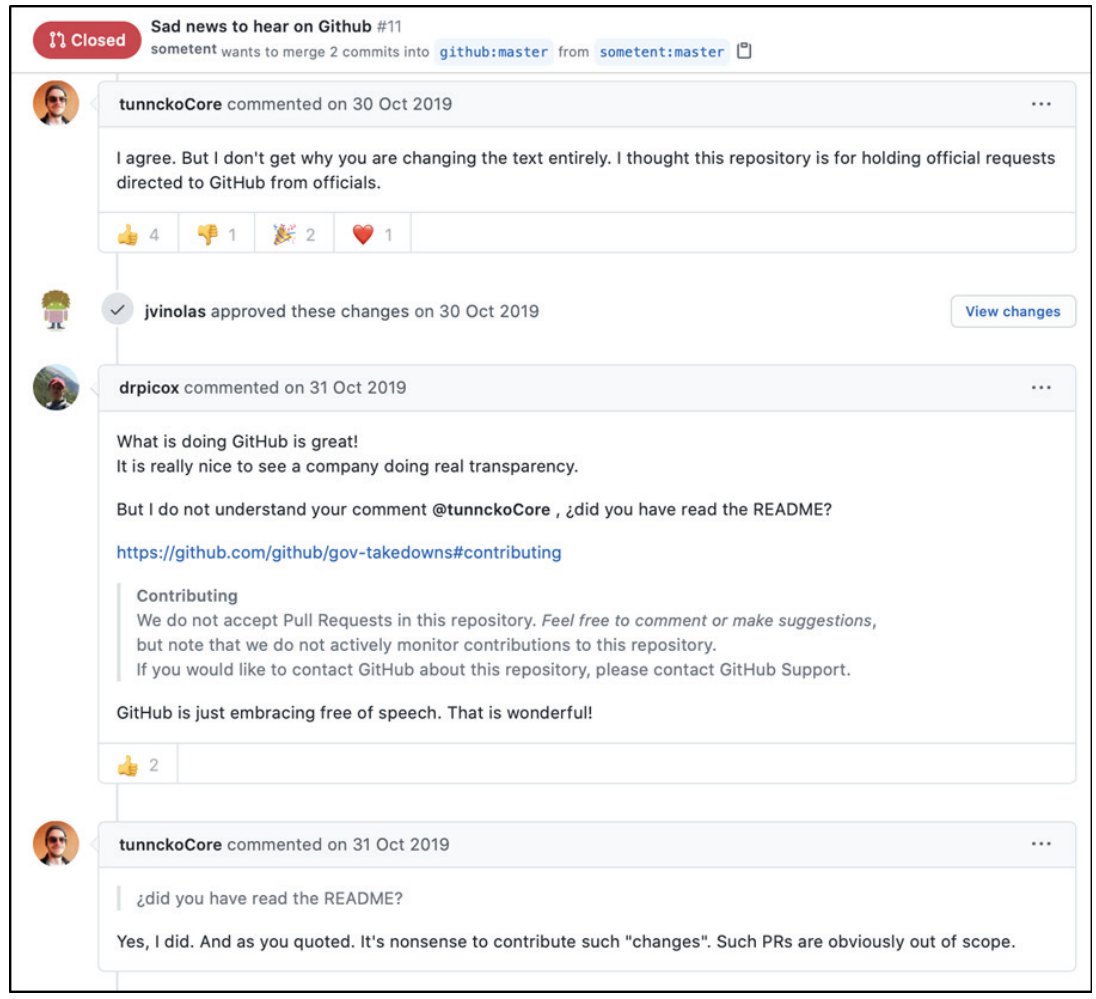

Figure 13. Exemple de discussion qui soulève l'inadéquation des participations politiques au script de la plateforme

La seconde manifestation de cette police normative, que l'on peut qualifier de « police technique », concerne des cas où les propriétaires de dépôts utilisent leur statut particulier pour stopper les échanges. GitHub, en tant que propriétaire du dépôt gov-takedowns, a d'abord refusé d'intégrer les PR proposées au dépôt, puis, pour 5 des $8 \mathrm{PR}$, l'entreprise a défini celles-ci comme étant « hors-sujet » et a restreint la possibilité de commenter ces PR aux seul·es collaborateur·rices de l'entreprise, empêchant ainsi toute nouvelle discussion de se tenir dans

27 https:/github.com/github/gov-takedowns/pull/9 
ces espaces (voir Figure 14). Ici, la défense du script de GitHub passe donc par le contrôle de la part des propriétaires des dépôts de qui a la possibilité d'écrire et de s'exprimer.

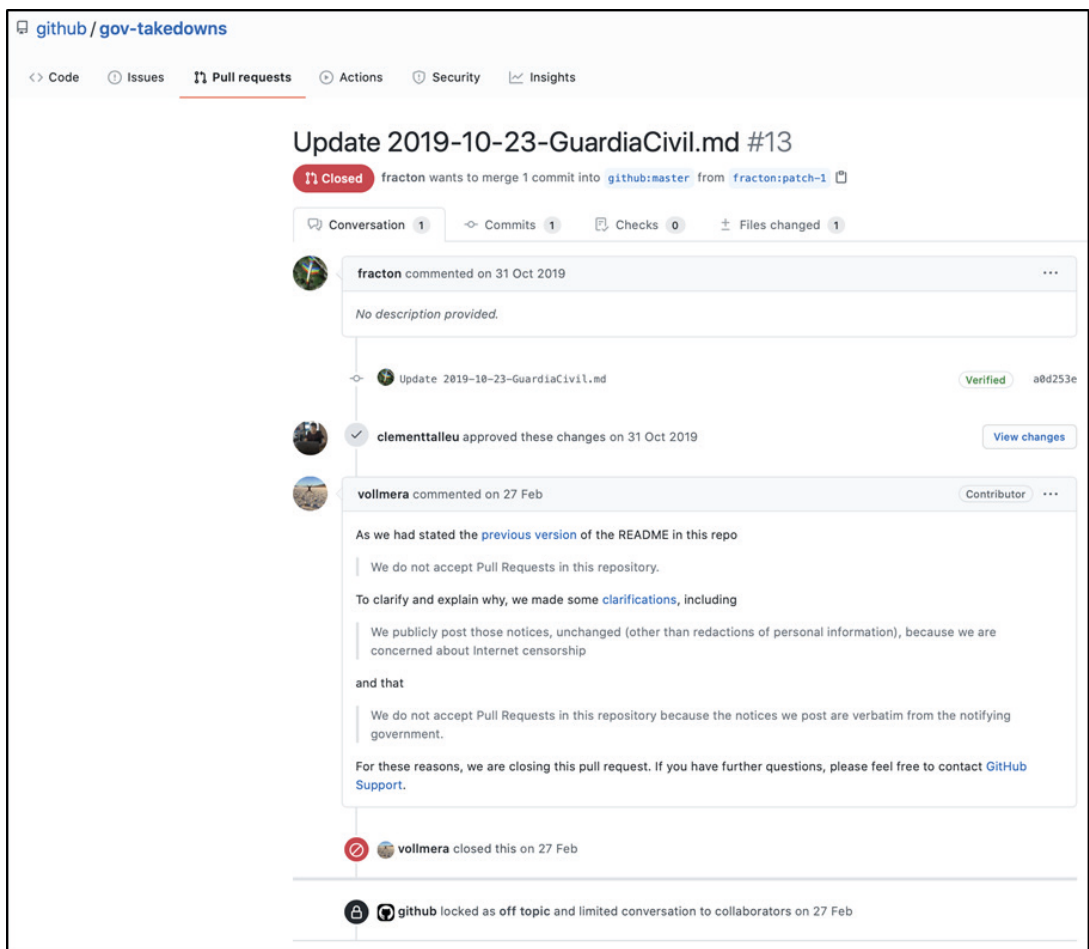

Figure 14. Restriction de participation imposée par Github

\section{Conclusion}

Peut-on vraiment considérer GitHub comme un tiers espace ? L'enquête dont nous avons rendu compte dans cet article permet de proposer une réponse nuancée à cette question. Dans une tradition expressiviste propre à l'espace public numérique (Monnoyer-Smith, 2011), les internautes s'emparent des TICN pour partager leurs opinions et construire de nouvelles modalités d'action collective en prenant appui sur les ressources communicationnelles de dispositifs tels que GitHub. Conformément aux évolutions de l'agir politique contemporain qui prend en compte notre capacité à embrasser des causes multiples qui constituent notre identité, les informaticien-nes présent·es sur GitHub 
se mobilisent sur différents sujets, de l'évolution de la vie parlementaire française à l'indépendantisme catalan, hybridant leur activisme avec la culture numérique dans laquelle ils baignent. En cela, la plateforme constitue un tiers espace, c'est-à-dire un lieu privilégié d'échange et de co-construction susceptible de produire du débat démocratique.

Pourtant, on observe que sur la plateforme des formes verticales de pouvoir perdurent et sont inscrites dans le script de la plateforme qui est d'abord et avant tout conçu pour produire le meilleur code informatique de manière efficace. Loin d'un idéal d'horizontalité, GitHub définit, pour chacun des dépôts, des hiérarchies entre les membres (propriétaire, membre, collaborateur.rice, contributeur-rice) et donne un pouvoir d'agir différent selon qui l'on est et selon le dépôt dans lequel on se trouve, tout ceci dans l'optique de « faire avancer le projet ». En conséquence, les participant·es se voient prescrire des formes de contribution et de participation situées en fonction de leur statut. Cette caractéristique de GitHub tourne largement à l'avantage des communautés les mieux implantées sur la plateforme. Les tentatives de débordement du script, de critiques des fichiers de codes informatiques et de textes qui sortent de l'ordinaire peuvent ainsi vite être contrôlées par des formes de «police normative » exercées par les acteurs en position dominante. Une nouvelle fois, en contexte numérique, le pouvoir s'exerce par le contrôle des architectures techniques et par la définition des capacités d'action des utilisateur rices.

\section{Références}

Akrich, M. (1987). Comment décrire les objets techniques? Techniques et culture, 9, 49-64. Doi : https://doi.org/10.4000/tc. 863

Badouard, R. (2014). La mise en technologie des projets politiques. Une approche « orientée design » de la participation en ligne. Participations, 8(1), 31-54. Doi : https://doi.org/10.3917/parti.008.0031

Badouard, R. (2017). Le Désenchantement de l'Internet : Désinformation, rumeur et propagande. Coll. Présence/Questions de société. Limoges, Fyp éditions.

Bellon, A. (2014). S'engager pour Internet : La mobilisation en ligne de la communauté informatique. Politiques de communication, 3, 151-175. Doi : https://doi. org $/ 10.3917 /$ pdc.003.0151

Bennett, L. \& Segerberg, A. (2012). The Logic of Connective Action: Digital Media and the Personalization of Contentious Politics. Information, Communication \& Society, 15(5), 739-768. Doi : https://doi.org/10.1080/1369118X.2012.670661

Broca, S. (2013). Utopie du logiciel libre : Du bricolage informatique à la réinvention sociale. Neuvy-en-Champagne, Le Passager clandestin.

Cardon, D. (2019). Culture numérique. Paris : Presses de Sciences Po. 
Cardon, D. \& Levrel, J. (2009). La vigilance participative. Une interprétation de la gouvernance de Wikipédia. Réseaux, 154(2), 51-89. Doi : https://doi.org/10.3917/ res. 154.0051

Couture, S. (2012). L'écriture collective du code source informatique : Le cas du commit comme acte d'écriture. Revue d'anthropologie des connaissances, 1(6), 21-42. Doi : https://doi.org/10.3917/rac.015.0061

Dabbish, L., Stuart, C., Tsay, J. \& Herbsleb, J. (2012). Social Coding in GitHub: Transparency and Collaboration in an Open Software Repository. Proceedings of the ACM 2012 Conference on Computer Supported Cooperative Work - CSCW '12, 1277-1286. Doi : https://doi.org/10.1145/2145204.2145396

Demazière, D., Horn, F. \& Zune, M. (2011). Ethnographie de terrain et relation d'enquête. Observer les « communautés » de logiciels libres. Sociologie, 2(2), 165-183. Doi : https://doi.org/10.3917/socio.022.0165

Gleizes, J. (2000). Introduction au logiciel libre. Multitudes, 1(1), 161-165. Doi : https://doi.org/10.3917/mult.001.0161

Graham T., Jackson D. \& Wright S. (2016). "We need to get Together and make ourselves heard": Everyday Online Spaces as Incubators of Political Action. Information, Communication \& Society, 10(19), 1373-1389. Doi : https://doi.org/10.1080/13691 18X.2015.1094113

Greffet, F. \& Wojcik, S. (2008). Parler politique en ligne : Une revue des travaux français et anglo-saxons. Réseaux, 150(4), 19-50.

Hirzalla, F., van Zoonen, L. \& de Ridder, J. (2011). Internet Use and Political Participation: Reflections on the Mobilization/Normalization Controversy. The Information Society, 27(1), 1-15. Doi : ttps://doi.org/10.1080/01972243.2011.534360

Jouët, J. (2000). Retour critique sur la sociologie des usages. Réseaux, 100(2), 487-521.

Jouët J. \& Le Caroff C. (2013). L'observation ethnographique en ligne. Dans C. Barats (éd.), Manuel d'analyse du Web en Sciences Humaines et Sociales (pp. 147-160). Coll. U. Paris : Armand Colin.

Lessig, L. (2000). Code Is Law. On Liberty in Cyberspace. Harvard Magazine. Disponible à : https://www.harvardmagazine.com/2000/01/code-is-law-html

Loveluck, B. (2015). Internet, une société contre l'État ? Libéralisme informationnel et économies politiques de l'auto-organisation en régime numérique. Réseaux, 192(4), 235-270. Doi : https://doi.org/10.3917/res.192.0235

Mabi, C. (2016). Décrypter le design des technologies numériques. Un enjeu pour une culture numérique plus réflexive ? Hybrid, 3. Disponible à : http://www.hybrid. univ-paris8.fr/lodel/index.php?id=634

Margolis, M. \& Resnick, D. (2000). Politics as Usual: The Cyberspace "Revolution ». Thousand Oaks : Sage Publications. Disponible à : http://dx.doi. org/10.4135/9781452233475

Monnoyer-Smith, L. (2011). La participation en ligne, révélateur d'une évolution des pratiques politiques ? Participations, 1(1), 156-185. doi : https://doi.org/10.3917/ parti.001.0156

Monnoyer-Smith, L. \& Wojcik, S. (2014). La participation politique en ligne, vers un renouvellement des problématiques ? Participations, 8(1), 5-29. Doi : https://doi. org/10.3917/parti.008.0005 
Oldenburg, R. (1989). The Great Good Place: Café, Coffee Shops, Community Centers, Beauty Parlors, General Stores, Bars, Hangouts, and How They Get You Through the Day. New York: Paragon House Publishers.

Paz, O. \& Savaroche, K. (2018). La communication comme négociation du sens : Le cas du développement informatique. Hermès, 3(82), 67-72. Doi : https://doi. org/10.3917/herm.082.0067

Shulz, S. (2019). Un logiciel libre pour lutter contre l'opacité du système sociofiscal : Sociologie d'une mobilisation hétérogène aux marges de l'État. Revue française de science politique, 69(5-6), 845-868. Doi : https://doi.org/10.3917/rfsp.695.0845

Thung, F., Bissyande, T. F., Lo, D. \& Lingxiao Jiang. (2013). Network Structure of Social Coding in GitHub. 2013 17th European Conference on Software Maintenance and Reengineering, 323-326.

Tolbert, C. J., Mossberger, K. \& McNeal, R. (2008). Institutions, Policy Innovation, and E-Government in the American States. Public Administration Review, 68(3), 549-563. Doi : https://doi.org/10.1111/j.1540-6210.2008.00890.x

Wright, S. (2012). From "Third Place" to "Third Space": Everyday Political Talk in Non-Political Online Spaces. Javnost - The Public. Journal of the European Institute for Communication and Culture, 19(3), 5-20. Doi : https://doi.org/10.1080/131 83222.2012.11009088

Wright, S. (2016). Les conversations politiques en ligne au quotidien : Design, délibération et « tiers espace ». Questions de communication, 30(2), 119-134. Doi : https:// doi.org/10.4000/questionsdecommunication.10734 


\section{(c) (1) $(9)$}

BY NC ND Publié sous la licence Creative Common

«Attibution - pas d'utilisation Commerciale - Pas de Modification 4.0. International» (CC BY-NC-ND) 